\title{
Three-Stage Turbo MBER Multiuser Beamforming Receiver Using Irregular Convolutional Codes
}

\author{
Shuang Tan, Jin Wang, Student Member, IEEE, Soon Xin Ng, Member, IEEE, \\ Sheng Chen, Senior Member, IEEE, and Lajos Hanzo, Fellow, IEEE
}

\begin{abstract}
Based on extrinsic information transfer (EXIT) charts, the convergence behavior of a three-stage serially concatenated multiuser beamforming receiver is presented. This system uses a linear minimum bit error rate (BER) multiuser detector as the inner module. Due to the nonrecursive nature of this inner module, a unity-rate memory-1 recursive precoder is placed in front of the channel to provide the required recursive structure. Irregular convolutional codes (IRCCs) are constructed to be used as the outer code to achieve near-capacity performance. Our simulations show that this system outperforms the traditional two-component iterative structure and is capable of significantly reducing the error floor.
\end{abstract}

Index Terms-Beamforming, extrinsic information transfer (EXIT) charts, irregular convolutional codes (IRCCs), minimum bit error rate (MBER).

\section{INTRODUCTION}

$\mathbf{S}$ INCE the invention of turbo codes in 1993 [1], the concatenated coding and iterative decoding, philosophy has been extended to other communication schemes, such as turbo equalization [2], [3], trellis-coded modulation [4], or turbo multiuser detection [5], [6]. To investigate the iterative detection process and its convergence, the powerful concept of semianalytical extrinsic information transfer (EXIT) charts was introduced in [7] and has been successfully applied in various concatenated schemes [6], [8]-[13].

It is widely recognized that in a serially concatenated code employing iterative decoding, the inner code should be recursive to maximize the attainable interleaver gain [14]. This benefit may be generalized to diverse serially concatenated schemes. However, not all inner modules of serially concatenated schemes can be readily rendered recursive, which, hence, limits the achievable decoding performance. In our system, a soft-in-soft-out (SISO) minimum bit error rate (MBER) multiuser detector (MUD) [15], [16] is invoked, which directly minimizes the bit error rate (BER) at the MUD's output rather than the mean square error (MSE) optimized by the classic minimum MSE (MMSE) detector. The nonrecursive nature

Manuscript received March 21, 2007; revised June 9, 2007, July 31, 2007, and August 1, 2007. This work was supported in part by the European Union under the auspices of the PHOENIX and NEWCOM projects and in part by the Engineering and Physical Sciences Research Council, U.K. The review of this paper was coordinated by Prof. X.-G. Xia.

The authors are with the School of Electronics and Computer Science, University of Southampton, SO17 1BJ Southampton, U.K. (e-mail: st104r@ ecs.soton.ac.uk; jw02r@ecs.soton.ac.uk; sxn@ecs.soton.ac.uk; sqc@ecs.soton. ac.uk; 1h@ecs.soton.ac.uk).

Digital Object Identifier 10.1109/TVT.2007.907296 of the MBER MUD may be ameliorated with the aid of a simple unity-rate memory-1 recursive precoder incorporated at the transmitter, and hence, the attainable performance may be further improved [17]. Then, at the receiver, the inner component composed of the MBER MUD, the intermediate channel decoder, and the outer channel decoder constitute a three-stage serially concatenated scheme.

In this paper, we design this three-stage concatenated multiuser receiver to achieve near-capacity performance [13]. By combining and projecting a series of 3-D EXIT functions onto a single 2-D EXIT chart [12], the convergence behavior of the system is visualized. Specifically, irregular convolutional codes (IRCCs) [8] are constructed, which are used as the outer code to solve the EXIT curve fitting problem in [9], i.e., that of minimizing the area of the EXIT chart's open tunnel, implying that the system becomes capable of approaching the achievable capacity.

The outline of this paper is given as follows: After introducing our system model in Section II, a novel MBER SISO interference canceller is derived in Section III. The system's convergence behavior is analyzed in Section IV with the aid of 3-D EXIT charts and their 2-D projections. Section V describes the design procedure of IRCCs. Our simulation results for this system communicating over both additive white Gaussian noise (AWGN) and Ricean flat-fading channels are presented in Section VI. Our conclusions are presented in Section VII.

\section{System Model}

The system supports $K$ binary phase-shift keying (BPSK) users, and each user transmits his/her signal through a single antenna on the same carrier frequency of $\omega=2 \pi f$. The receiver is equipped with a linear antenna array consisting of $L$ elements, which have a uniform element spacing of $\lambda / 2$, where $\lambda$ is the wavelength. Then, the signal samples received at the symbol rate can be expressed as $r_{l}(i)=\sum_{k=1}^{K} A_{k}(i) s_{k}(i) e^{j \omega t_{l}\left(\theta_{k}\right)}+$ $n_{l}(i)$ for $1 \leq l \leq L$, where $s_{k}(i)$ is the $i$ th symbol of the $k$ th BPSK user, $A_{k}(i)$ is the complex-valued channel coefficient when transmitting the $i$ th symbol of user $k, n_{l}(i)$ is the complex-valued Gaussian white noise having a power of $2 \sigma_{n}^{2}$, and $t_{l}\left(\theta_{k}\right)=(\lambda / 2)(l-1) \sin \left(\theta_{k}\right) / c$ is the relative time delay at array element $l$ for the source signal of user $k$, with $\theta_{k}$ being the line of sight angle of arrival for source $k$, and $c$ is the speed of light. The complex-valued system vector $\mathbf{h}_{k}=\left[A_{k} e^{j \omega t_{1}\left(\theta_{k}\right)} A_{k} e^{j \omega t_{2}\left(\theta_{k}\right)}, \ldots, A_{k} e^{j \omega t_{l}\left(\theta_{k}\right)}\right]^{T}$ constitutes the unique user-specific signature of user $k$. In this paper, we assume that the relative time delay of all users with respect 


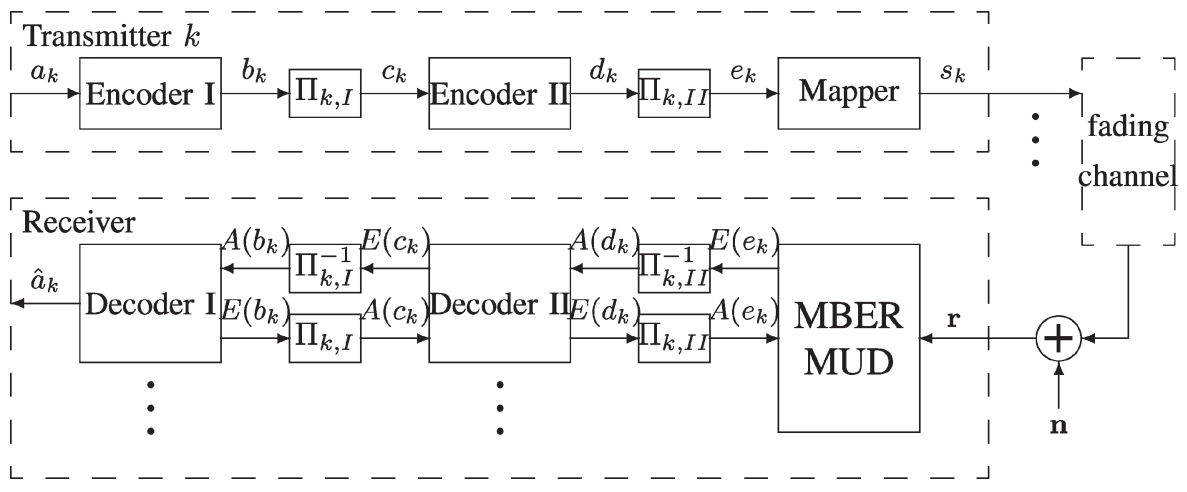

Fig. 1. Three-stage iterative multiuser beamforming system structure.

to the angularly closest neighbors is the same. All the angular locations of the users were selected under this constraint.

Fig. 1 depicts the system structure of the proposed threestage serially concatenated multiuser communication system. In this figure, only one transmitter and the $k$ th user's receiver were portrayed. At transmitter $k$, a block of information bits $a_{k}$ is first encoded by channel encoder I. Then, the outer encoded bits $b_{k}$ are interleaved, yielding the permuted bits $c_{k}$, which are fed through the unity-rate memory-1 encoder II. The resultant "double-encoded" bits $d_{k}$ are interleaved by a second interleaver, yielding the interleaved bits $e_{k}$, which are fed to a bit-to-modulated-symbol mapper, as seen in Fig. 1. After mapping, the modulated signal $s_{k}$ is transmitted over a memoryless AWGN or fading channel. At the receiver in Fig. 1, an iterative MUD/decoding structure is employed, where extrinsic information is exchanged between the three SISO modules, namely the MBER MUD, the a posteriori probability (APP)-based decoder II, and the APP-based decoder $\mathrm{I}^{1}{ }^{1}$ in a number of consecutive iterations. To be specific, in Fig. 1, $A(\cdot)$ denotes the a priori information that is represented in terms of log-likelihood ratios (LLRs) [18], while $E(\cdot)$ denotes the extrinsic information that is also expressed in terms of LLRs. Note that decoder II processes two a priori inputs arriving from both the MBER MUD and decoder I to generate two extrinsic outputs. Following the last iteration, the estimates $\hat{a}_{k}$ of the original bits are generated by decoder I, as seen in Fig. 1.

\section{MBER SISO INTERFERENCE CANCELLATION}

Let $\mathbf{w}_{k}$ denote the beamformer's weight vector for user $k$. When using the soft interference cancellation principle, the estimated complex-valued symbol of user $k$ can be expressed as

$$
\hat{s}_{k}=\mathbf{w}_{k}^{H}\left(\mathbf{r}-\mathbf{H} \overline{\mathbf{s}}+\bar{s}_{k} \mathbf{h}_{k}\right)
$$

where $\mathbf{H}=\left[\mathbf{h}_{1} \mathbf{h}_{2}, \ldots, \mathbf{h}_{K}\right], \overline{\mathbf{s}}=\left[\bar{s}_{1} \bar{s}_{2}, \ldots, \bar{s}_{K}\right]^{T}$, and $\bar{s}_{k}$ is the real-valued mean of the $k$ th user's symbol based on the a priori information.

\footnotetext{
${ }^{1}$ Each user has a separate decoder I, decoder II, interleavers, and deinterleavers.
}

In BPSK MBER beamforming-aided systems supporting $K$ users, the error probability is given by [15]

$$
\operatorname{Pe}_{k}\left(\mathbf{w}_{k}\right)=\sum_{q=1}^{2^{K}} P\left(\mathbf{s}^{(q)}\right) \cdot Q\left[\frac{\operatorname{sgn}\left(\Re\left[s_{k}^{(q)}\right]\right) \cdot \Re\left[\overline{\hat{s}}_{k}^{(q)}\right]}{\sigma_{n} \sqrt{\mathbf{w}_{k}^{H} \mathbf{w}_{k}}}\right]
$$

where $P\left(\mathbf{s}^{(q)}\right)=\prod_{k} P\left(s_{k}=s_{k}^{(q)}\right)$ is the a priori probability of transmitting the $q$ th possible symbol combination $\mathbf{s}^{(q)}$ of the $K$ users, $\overline{\hat{s}}_{k}^{(q)}=\mathbf{w}_{k}^{H}\left(\mathbf{H} \mathbf{s}^{(q)}-\mathbf{H} \overline{\mathbf{s}}+\bar{s}_{k} \mathbf{h}_{k}\right)$ is the $k$ th user's estimated symbol ignoring the effects of noise, and $Q[x]=$ $(1 / \sqrt{2 \pi}) \int_{-\infty}^{x} e^{-t^{2} / 2} d t$. The MBER beamforming solution derived for BPSK is then defined as

$$
\mathbf{w}_{k}=\arg \min _{\mathbf{w}} \operatorname{Pe}_{k}\left(\mathbf{w}_{k}\right)
$$

This MBER beamforming weight optimization problem can be solved using the simplified conjugate gradient algorithm, which is detailed in [15], formulating the gradient of the bit error probability as follows:

$$
\begin{aligned}
\nabla \operatorname{Pe}_{k}\left(\overline{\mathbf{w}}_{k}\right) & =\frac{1}{\sqrt{2 \pi} \sigma_{n}} \sum_{q=1}^{2^{K}} P\left(\mathbf{s}^{(q)}\right) \\
& \cdot \exp \left(-\frac{\left.\left(\Re\left[\overline{\hat{s}}_{k}^{(q)}\right]\right)^{2}\right)}{2 \sigma_{n}^{2}}\right) \operatorname{sgn}\left(\Re\left[s_{k}^{(q)}\right]\right) \\
& \cdot\left(\overline{\mathbf{w}}_{k} \Re\left[\overline{\hat{s}}_{k}^{(q)}\right]-\left(\mathbf{H} \mathbf{s}^{(q)}-\mathbf{H} \overline{\mathbf{s}}+\bar{s}_{k} \mathbf{h}_{k}\right)\right)
\end{aligned}
$$

where $\overline{\mathbf{w}}_{k}$ is the unity-norm normalized version of vector $\mathbf{w}_{k}$.

The MBER MUD's output extrinsic information cannot be calculated in the same way as that of the MMSE solution in [5], since the conditional probability density function $p\left(\hat{s}_{k} \mid s_{k}=\right.$ $\left.s_{k}^{(q)}\right)$ of the MBER MUD's output signal is non-Gaussian. However, we can assume that $\Re\left[\hat{s}_{k}\right]$, which is the real part of the output signal, is approximately 1-D Gaussian distributed. Then, the output LLR can be expressed as

$$
E\left(e_{k}\right)=\frac{2 \Re\left[\hat{s}_{k}\right] \Re\left[\mathbf{w}_{k}^{H} \mathbf{h}_{k}\right]}{\Re\left[\mathbf{w}_{k}^{H} \mathbf{H}\right] \underline{\mathbf{V}}_{k} \Re\left[\mathbf{H}^{H} \mathbf{w}_{k}\right]+\sigma_{n}^{2} \mathbf{w}_{k}^{H} \mathbf{w}_{k}}
$$

where $\underline{\mathbf{V}}_{k}=\operatorname{diag}\left[v_{1}, \ldots, v_{k-1} \quad 0 \quad v_{k+1}, \ldots, v_{k}\right]$, and $v_{k}=$ $E_{s}-\left|\bar{s}_{k}\right|^{2}$. 


\section{Convergence Analysis Using EXIT Charts}

The EXIT chart analysis computes the mutual information (MI) between the LLRs and the corresponding bits, as detailed in [7]. Let $I_{A(x)}$ denote the MI between the a priori values $A(x)$ and bit sequence $x$. Furthermore, let $I_{E(x)}$ denote the MI between the extrinsic values $E(x)$ and the bit sequence $x$. The EXIT function of decoder I is defined as ${ }^{2}$

$$
I_{E(b)}=F_{d_{I}}\left(I_{A(b)}\right) .
$$

As seen in Fig. 1, decoder II has two extrinsic MI outputs, namely $I_{E(c)}$ and $I_{E(d)}$, both of which are functions of the a priori MI inputs $I_{A(c)}$ and $I_{A(d)}$. The two EXIT functions are defined as [12]

$$
\begin{aligned}
& I_{E(c)}=F_{d_{\mathrm{II}}, o}\left(I_{A(c)}, I_{A(d)}\right) \\
& I_{E(d)}=F_{d_{\mathrm{II}}, i}\left(I_{A(c)}, I_{A(d)}\right) .
\end{aligned}
$$

Unlike in the single-user turbo coding or turbo equalization, in the multiuser detection scenario, the MUD's EXIT curve recorded for the desired user depends on all the other $(K-1)$ users' channel decoder output MI, which implies that the MUD's EXIT surface should be $K$-dimensional. Note that for the MUD, the received signal has to be recorded for a given channel state and signal-to-noise ratio (SNR), because the MUD's received signal is affected by the channel quality that is quantified here in terms of the noise power. Then, the EXIT function of the MUD for user $k$ is defined as

$$
I_{E\left(e_{k}\right)}=F_{m, k}\left(\forall I_{A\left(e_{k^{\prime}}\right)}, E_{b} / N_{0}\right)
$$

where $1 \leq k^{\prime} \leq K$, and $k^{\prime} \neq k$. In our simulations, all users' SNRs were identical. Additionally, their angular locations were selected so that the relative time delay of all users with respect to the angularly closest neighbors was the same, as defined in Section II. Hence, the turbo MUD can average all the users' MIs to simplify the EXIT chart function (9) to

$$
I_{E(e)}=F_{m}\left(I_{A(e)}, E_{b} / N_{0}\right) .
$$

Consider a half-rate nonsystematic convolutional (NSC) code using the octally represented generators $(15,17)$ as encoder I and a simple rate- 1 accumulator as encoder II, which are described by the octal generator polynomials of (1/3), where 3 represents the feedback polynomial. All the EXIT functions can be plotted in two 3-D EXIT charts: one for EXIT functions (8) and (10), as shown in Fig. 2(a), and another for EXIT functions (6) and (7), as shown in Fig. 2(b). The intersection of the surfaces seen in Fig. 2(a) characterizes the best possible attainable performance when exchanging information between the MUD and decoder II after an infinite number of iterations at different fixed values of $I_{A(c)}$, which is shown as a thick solid line. For each point $\left[I_{A(c)}, I_{A(d)}, I_{E(d)}\right]$ of this line in the 3-D space in Fig. 2(a), there is a specific value of $I_{E(c)}$ determined by $I_{A(c)}$ and $I_{A(d)}$ according to EXIT function (7).

\footnotetext{
${ }^{2}$ Each user employs the same channel coding I and channel coding II schemes; hence, the subscript " $k$ " can be omitted in the EXIT functions of decoders I and II
}

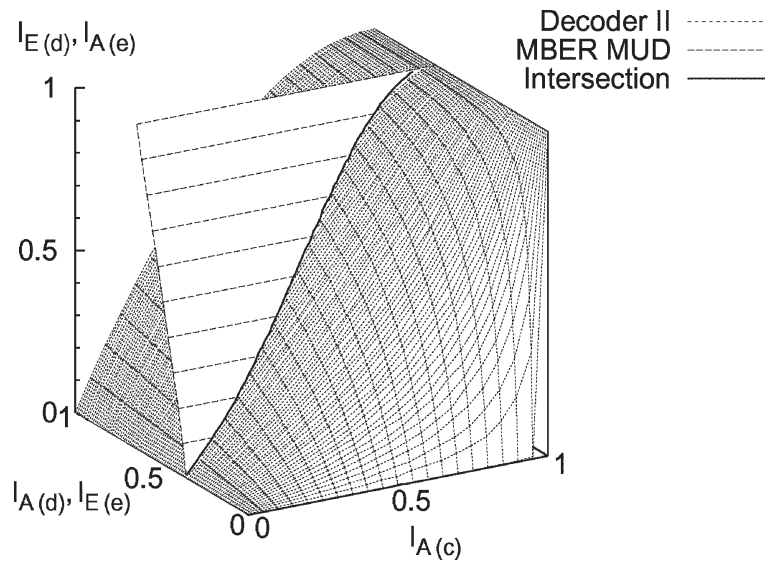

(a)

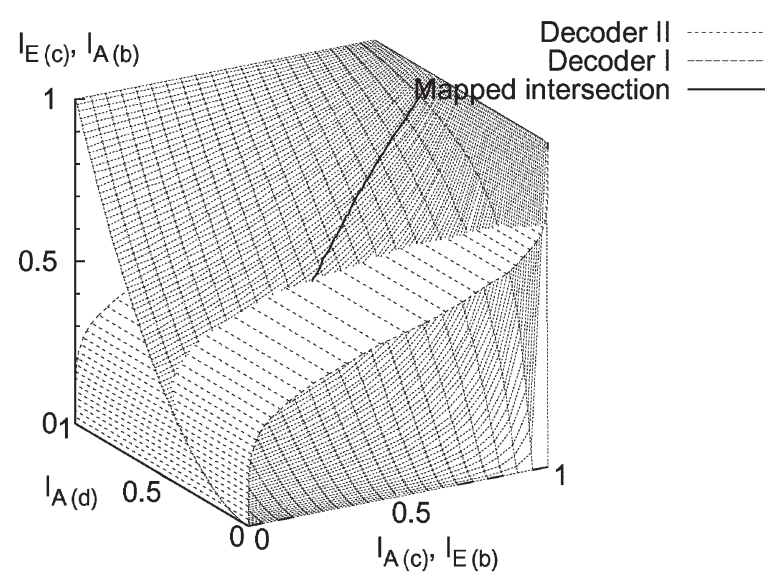

(b)

Fig. 2. Three-dimesional EXIT charts of the three-stage iterative MBER beamforming receiver supporting $K=6$ users with the aid of two antennas at $E_{b} / N_{0}=2 \mathrm{~dB}$. (a) Decoder II and MBER MUD at $E_{b} / N_{0}=2 \mathrm{~dB}$. (b) Decoders I and II.

Therefore, the solid line on the surface of the EXIT function of decoder II seen in Fig. 2(a) is mapped to the solid line shown in Fig. 2(b). To avoid the cumbersome 3-D representation, we now project the bold EXIT curve in Fig. 2(b) onto the 2-D plane at $I_{A(d)}=0$, yielding the solid line in Fig. 3 [12]. The EXIT curves of the MBER MUD recorded at $E_{b} / N_{0}=2 \mathrm{~dB}$ and that of decoder I are also plotted in Fig. 3. The projected EXIT curve can be described as

$$
I_{E(c)}=F_{p}\left(I_{A(c)}, E_{b} / N_{0}\right)
$$

which implies that the MUD and decoder II are combined into a single inner decoder component, and hence, the resultant threestage iterative scheme can be analyzed as a traditional two-stage iterative arrangement.

As for a traditional two-stage turbo scheme, the EXIT curve of the inner component and that of the outer component are used to analyze the associated convergence behavior. As seen in Fig. 3, the EXIT curve of the MBER MUD cannot reach the convergence point of $[1,1]$ and intersects with the EXIT curve of the outer NSC code, which implies that residual errors persist, regardless of both the number of iterations used and the size of the interleaver. This explains the residual BER encountered by the traditional two-stage turbo scheme. On the 


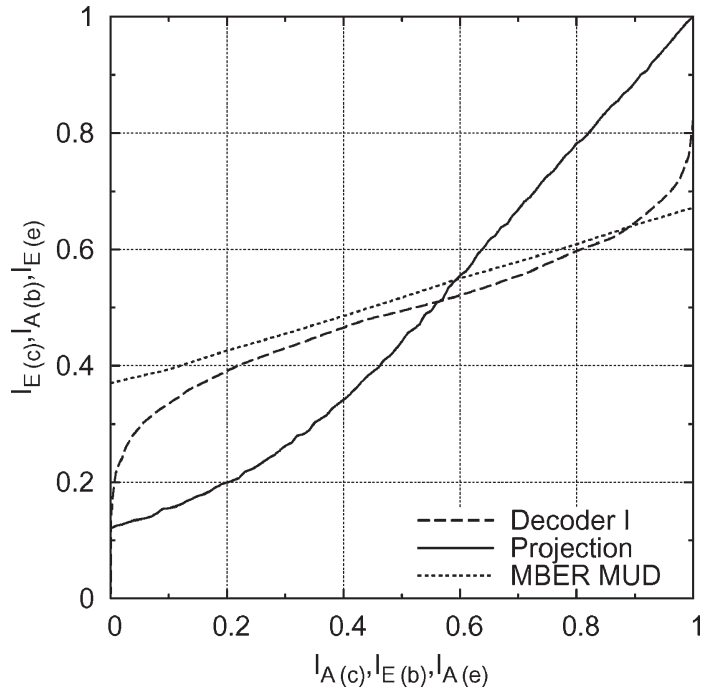

Fig. 3. Two-dimesional projected EXIT charts of the three-stage iterative MBER beamforming receiver supporting $K=6$ users with the aid of two antennas at $E_{b} / N_{0}=2 \mathrm{~dB}$.

other hand, if the inner MBER MUD and the intermediate decoder II are viewed as a single joint inner SISO module, then the projected EXIT function (11) is the EXIT function of this module, which is capable of reaching the convergence point of $[1,1]$. As long as there is an open tunnel between the EXIT curve of this joint inner module and that of the outer decoder, the three-stage concatenated system is capable of converging, hence achieving an infinitesimally low BER.

\section{DESIGN OF IRCC MBER BEAMFORMING}

According to the area properties [8], [9] of the EXIT charts, the area under the EXIT curve of the inner module is approximately equal to the channel capacity attained when the channel's input is uniformly distributed. Furthermore, the area under the EXIT curve of the outer code is approximately equal to $1-R_{I}$, where $R_{I}$ is the outer code rate. More explicitly, let $A_{d_{I}}$ and $\bar{A}_{d_{I}}$ be the areas under $F_{d_{I}}(i)$ and its inverse $F_{d_{I}}^{-1}(i), i \in[0,1]$, respectively. Similarly, we define $A_{m}$ for $F_{m}\left(i, E_{b} / N_{0}\right)$ and $A_{p}$ for $F_{p}\left(i, E_{b} / N_{0}\right)$. Then, we have $\bar{A}_{d_{I}} \approx$ $R_{I}$, and for BPSK modulation, $A_{m} \approx C$, where $C$ is the capacity of the communication channel when its input is uniformly distributed. Since the intermediate channel code II has a unity rate, the area $A_{p}$ under the projected EXIT curve is also approximately equal to the aforementioned uniform-input channel capacity $C$. These area properties yield a design rule for our system: $R_{I}$ should approach $C$ as closely as possible under the constraint $F_{d_{I}}^{-1}(i)<F_{p}\left(i, E_{b} / N_{0}\right), \forall i \in[0,1)$, implying that an outer code is sought, which ensures that the inverted EXIT curve $F_{d_{I}}^{-1}(i)$ fits $F_{p}\left(i, E_{b} / N_{0}\right)$ as closely as possible, while maintaining an open EXIT tunnel and, hence, minimizing the area of the open EXIT tunnel.

However, as seen in Fig. 3, even if the area under the projected EXIT curve that is plotted using the continuous line at $E_{b} / N_{0}=2 \mathrm{~dB}$ is $A_{p} \approx 0.51$, which is larger than the outer code rate of $R_{I}=0.5$, no open EXIT tunnel exists. To circumvent this problem, we introduce the novel concept of IRCCs

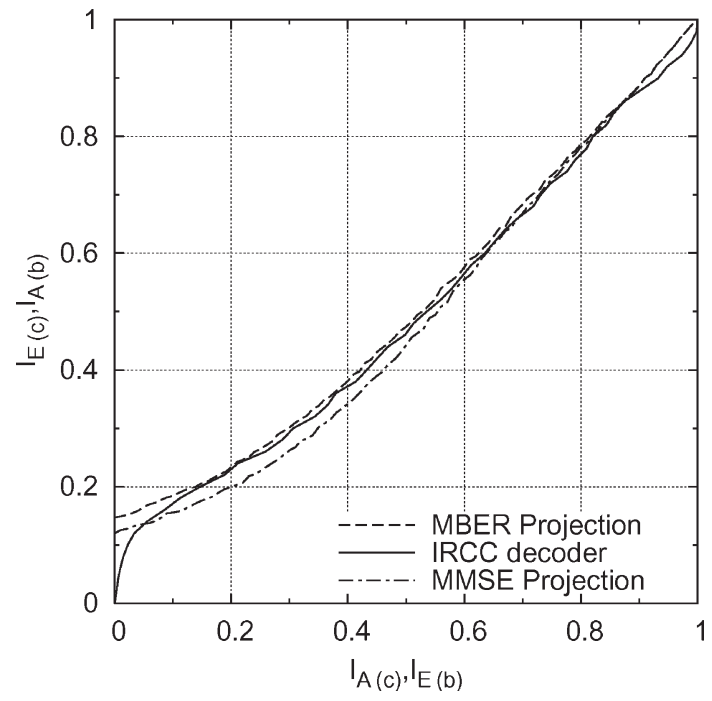

Fig. 4. Projected and optimized EXIT curves of the IRCC for the three-stage iterative beamforming receiver supporting $K=6$ users with the aid of two antennas at $E_{b} / N_{0}=2 \mathrm{~dB}$.

[8], which allows us to shape the outer code's EXIT curve to match that of the inner code. IRCCs were specifically designed with the aid of EXIT charts to improve the convergence behavior of iteratively decoded systems and are constituted by a superposition of convolutional codes having different code rates. To be specific, an IRCC is constructed from a family of $P$ subcodes. Each subcode has its own individual code rate $r_{p}(1 \leq p \leq P)$ and encodes a specific fraction of $\alpha_{p} r_{p} N$ of the original uncoded information bits, hence generating $\alpha_{p} N$ number of encoded bits, where $N$ denotes the total number of encoded bits. Given the target overall average code rate of $R \in$ $[0,1]$, the weighting coefficient $\alpha_{p}$ has to satisfy $\sum_{p=1}^{P} \alpha_{p}=1$, $\sum_{p=1}^{P} \alpha_{p} r_{p}=R$, and $\alpha_{p} \in[0,1]$. Clearly, the individual code rates $\left\{r_{p}\right\}$ and the weighting coefficients $\left\{\alpha_{p}\right\}$ play crucial roles in shaping the EXIT function of the resultant IRCC. The EXIT function $F_{d_{I}}\left(I_{A}\right)$ of the target IRCC is the weighted superposition of its subcodes' EXIT functions $F_{d_{I}, p}\left(I_{A}\right)$ [8], yielding

$$
F_{d_{I}}\left(I_{A}\right)=\sum_{p=1}^{P} \alpha_{p} F_{d_{I}, p}\left(I_{A}\right) .
$$

By using the 17 subcodes and optimization criterion 2 introduced in [8], we now optimize the weighting coefficients $\left\{\alpha_{p}\right\}$, so that the IRCC's EXIT curve matches the projected EXIT curve. Fig. 4 shows the target projected MBER EXIT curve and the resultant EXIT curve of the optimized IRCC at $E_{b} / N_{0}=2 \mathrm{~dB}$. The area under the projected MBER EXIT curve at $E_{b} / N_{0}=2 \mathrm{~dB}$ is $A_{p} \approx 0.51$, which indicates that this $E_{b} / N_{0}$ value is close to the lowest possible convergence threshold for a system having an outer coding rate of $R_{I}=0.5$. Despite the fact that $A_{p}$ and $R_{I}$ are so close to each other, there is still an open tunnel between the two curves in Fig. 4, which explicitly indicates the flexibility of the IRCCs. Fig. 4 compares the projected MMSE EXIT curve and that of the MBER MUD, where the area under the MMSE curve is about 0.48. This 


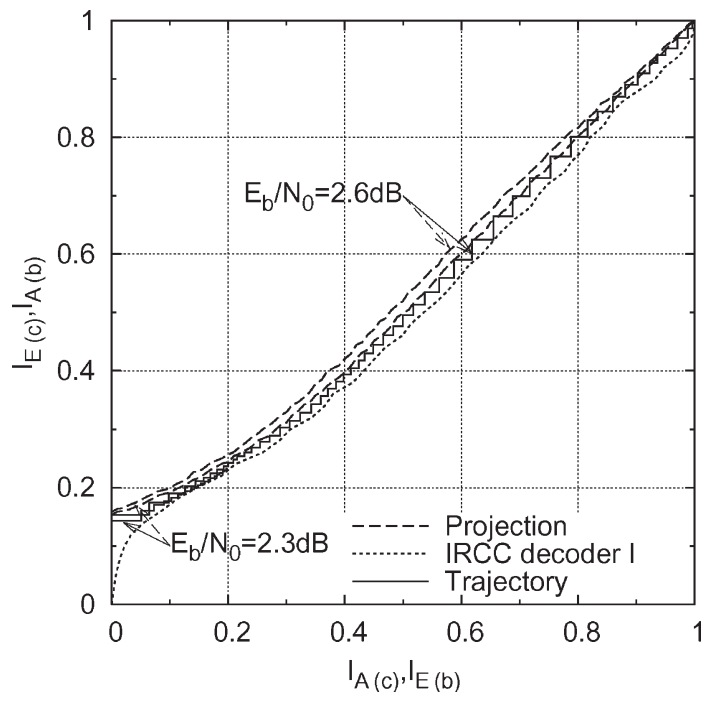

Fig. 5. EXIT charts and recorded iterative trajectory for the three-stage iterative MBER beamforming receiver using IRCCs to support $K=6$ users with the aid of two antennas at $E_{b} / N_{0}=2.3$ and $2.6 \mathrm{~dB}$.

implies that the MMSE receiver needs a higher SNR to maintain an open tunnel.

\section{Simulation Results}

The system employs a two-element receive antenna array. All $K=6$ users employ BPSK modulation and have the same transmit power. ${ }^{3}$ The angular separations of users with respect to the antenna array are $15^{\circ},-24^{\circ}, 68^{\circ},-4^{\circ}, 36^{\circ}$, and $-48^{\circ}$. Each user employs two different randomly generated interleavers having a length of $2 \times 10^{4}$ bits. The code rate of encoder I is 0.5 .

When communicating over AWGN channels, the iterative decoding trajectories recorded during our Monte Carlo simulations using the optimized IRCC at $E_{b} / N_{0}=2.3$ and $2.6 \mathrm{~dB}$ are depicted in Fig. 5. It can be seen that when $E_{b} / N_{0}=2.6 \mathrm{~dB}$, the recorded trajectory converges to the $\left[I_{A(c)}, I_{E(c)}\right]=[1,1]$ point through the predicted EXIT chart tunnel. Furthermore, since the tunnel between the two EXIT curves is very narrow, a significant number of iterations are required to enable the iterative receiver to converge to the point of $[1,1]$.

Fig. 6 shows the BER performance of the three-stage iterative beamforming scheme using IRCCs and the conventional twostage system using an NSC code having a constraint length of 4 and octal generator polynomials of $(15,17)$ as the outer code. It can be seen in Fig. 6 that the two-stage MBER receiver exhibits an error floor, which corresponds to the BER performance of the single-user case. By contrast, the three-stage MBER and MMSE system becomes capable of achieving an infinitesimally low BER when the $E_{b} / N_{0}$ encountered is higher than 2.5 and $3.5 \mathrm{~dB}$, respectively. This BER figure confirms the performance difference of the two MUDs, which we have predicted from the EXIT curves in Fig. 4.

\footnotetext{
${ }^{3}$ Please note that this is a highly rank-deficient system, since the number of transmit antennas of the users supported is three times higher than the number of receive antennas. Hence, conventional MMSE receivers would exhibit a high residual BER, even for $K=3$ users.
}

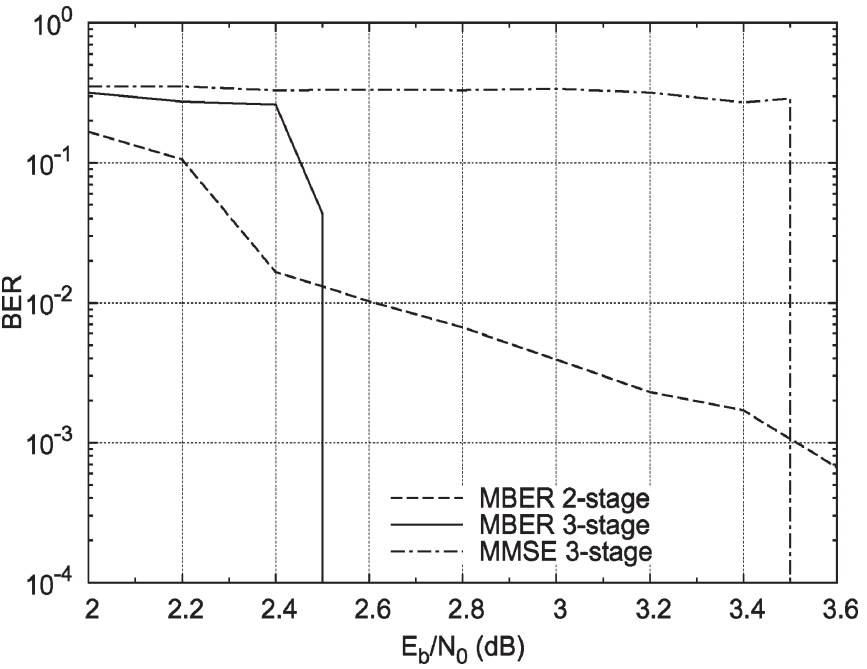

Fig. 6. BER performance of the three- and two-stage iterative beamforming receivers supporting $K=6$ users with the aid of two antennas.

We observe in Fig. 4 that the three-stage iterative system is capable of maintaining an open tunnel and, hence, of converging to the point of $[1,1]$ in the EXIT chart at $E_{b} / N_{0}=$ $2 \mathrm{~dB}$. However, the $E_{b} / N_{0}$ threshold of the three-stage system characterized in Fig. 6 is $2.6 \mathrm{~dB}$. When $E_{b} / N_{0}=2.3 \mathrm{~dB}$, we can see the formation of an open tunnel in Fig. 5. However, the actual decoding trajectory aborts at a point around [0.12, 0.19] for the following reasons.

1) Our system is a multiuser system. Although we selected the arrival angles of the users' signals under the rule defined in Section II, the achievable performance of the users is still not perfectly identical. In the EXIT charts, we used the average MUD EXIT curve and trajectory. When the average EXIT tunnel is quite narrow, a certain user's tunnel may, in fact, be closed. This poor performance of a single user may render the parallel interference cancellation mechanism that is unable to remove the multiuser interference.

2) In our simulations, the activation order of the consecutive iterations is "MUD, decoder II, decoder I, decoder II, MUD, ...." There is only one iteration between the MUD and decoder II before the extrinsic information is transferred to decoder I. It implies that the accurate 2-D projected curve recorded for this particular activation order of the decoders should be a little lower than the projected curve seen in Fig. 5, and the actual tunnel may become narrower or even closed.

3) The projected curve is the mapped intersection of the MUD's EXIT surface and the decoder II's EXIT surface. It represents the best possible output MI of the combined component. However, the practically achievable performance may be worse, potentially leading to a lower projected 2-D curve and a higher SNR threshold.

Let us now consider the aforementioned three-stage system's convergence performance when communicating over Ricean flat-fading channels. The normalized Doppler frequency is 0.01 , and the Ricean $K$-factor is 10 . Our simulation results demonstrate that the $E_{b} / N_{0}$ threshold required to achieve an 


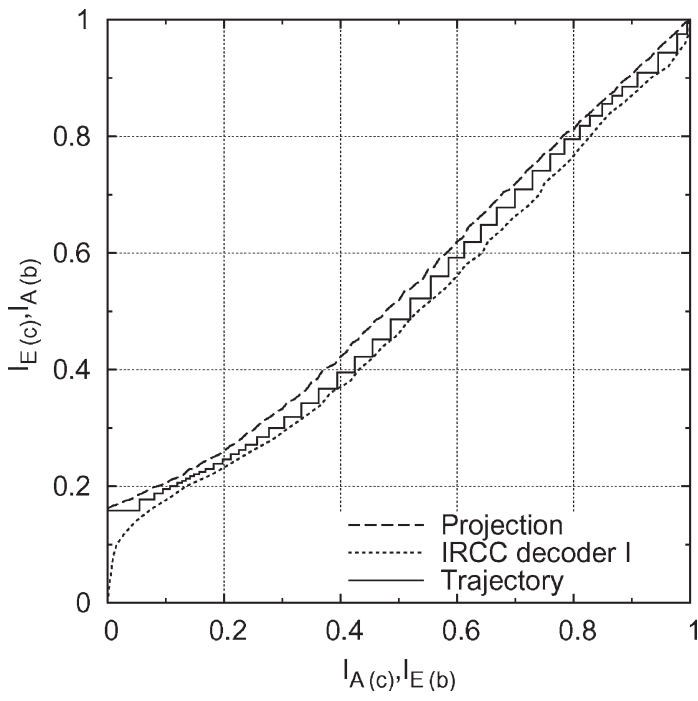

Fig. 7. EXIT charts and recorded iterative trajectory for the three-stage iterative MBER beamforming receiver using IRCCs to support $K=6$ users with the aid of two antennas at $E_{b} / N_{0}=3.0 \mathrm{~dB}$ over Ricean flat-fading channels.

infinitesimally low BER is $3.0 \mathrm{~dB}$. At this SNR, the recorded iterative decoding trajectory and the resultant EXIT curves are shown in Fig. 7.

\section{CONCLUSION}

In this paper, we have proposed a three-stage serial concatenated multiuser system, where the inner SISO MBER MUD module cannot be rendered recursive. Hence, the iterative receiver was extended to the following three SISO modules: 1) the inner MBER MUD; 2) the intermediate unity-rate channel decoder; and 3) the outer channel decoder. Furthermore, the convergence behavior of our design example was analyzed using 3-D EXIT charts and their 2-D projections. It has been pointed out that although the EXIT function of the inner MBER MUD cannot reach the point of perfect convergence at $[1,1]$, the joint EXIT function obtained for the amalgamated inner MBER MUD and the intermediate channel decoder with the aid of projection can. Therefore, the three-stage system is capable of eliminating the residual BER encountered in the conventional two-stage system. With the advent of 2-D projection, an IRCC was constructed for employment as the outer code, whose EXIT function was matched to the joint EXIT function, and as a result, the channel capacity was closely approached.

\section{REFERENCES}

[1] C. Berrou, A. Glavieux, and P. Thitimajshima, "Near Shannon limit errorcorrecting coding and decoding: Turbo-codes," in Proc. IEEE Int. Conf. Commun., Geneva, Switzerland, vol. 2, May 1993, pp. 1064-1070.

[2] M. Tüchler, A. C. Singer, and R. Koetter, "Minimum mean squared error equalization using a priori information," IEEE Trans. Signal Process., vol. 50, no. 3, pp. 673-683, Mar. 2002.

[3] M. Tüchler, R. Koetter, and A. C. Singer, "Turbo equalization: Principles and new results," IEEE Trans. Commun., vol. 50, no. 5, pp. 754-767, May 2002

[4] X. Li and J. Ritcey, "Trellis-coded modulation with bit interleaving and iterative decoding," IEEE J. Sel. Areas Commun., vol. 17, no. 4, pp. 715724, Apr. 1999.
[5] X. Wang and H. V. Poor, "Iterative (turbo) soft interference cancellation and decoding for coded CDMA," IEEE Trans. Commun., vol. 47, no. 7, pp. 1046-1060, Jul. 1999.

[6] A. Tarable, G. Montorsi, and S. Benedetto, "A linear front end for iterative soft interference cancellation and decoding in coded CDMA," IEEE Trans. Wireless Commun., vol. 4, no. 2, pp. 507-518, Mar. 2005.

[7] S. ten Brink, "Convergence behavior of iteratively decoded parallel concatenated codes," IEEE Trans. Commun., vol. 40, no. 10, pp. 1727-1737, Oct. 2001.

[8] M. Tüchler, "Design of serially concatenated systems depending on the block length," IEEE Trans. Commun., vol. 52, no. 2, pp. 209-218, Feb. 2004.

[9] A. Ashikhmin, G. Kramer, and S. ten Brink, "Extrinsic information transfer functions: Model and erasure channel properties," IEEE Trans. Inf. Theory, vol. 50, no. 11, pp. 2657-2673, Nov. 2004.

[10] K. Li and X. Wang, "EXIT chart analysis of turbo multiuser detection," IEEE Trans. Wireless Commun., vol. 4, no. 1, pp. 300-311, Jan. 2005.

[11] J. Hou, P. Siegel, and L. Milstein, "Design of multi-input multi-output systems based on low-density parity-check codes," IEEE Trans. Commun., vol. 53, no. 4, pp. 601-611, Apr. 2005.

[12] F. Brännström, L. K. Rasmussen, and A. J. Grant, "Convergence analysis and optimal scheduling for multiple concatenated codes," IEEE Trans. Inf. Theory, vol. 51, no. 9, pp. 3354-3364, Sep. 2005.

[13] J. Wang, S. X. Ng, A. Wolfgang, L. L. Yang, S. Chen, and L. Hanzo, "Near-capacity three-stage MMSE turbo equalization using irregular convolutional codes," in Proc. Turbo-Coding, Munich, Germany, 2006. Electronic publication.

[14] S. Benedetto, D. Divsalar, G. Montorsi, and F. Pollara, "Serial concatenation of interleaved codes: Performance analysis, design, and iterative decoding," IEEE Trans. Inf. Theory, vol. 44, no. 3, pp. 909-926, May 1998.

[15] S. Chen, N. N. Ahmad, and L. Hanzo, "Adaptive minimum bit-error rate beamforming," IEEE Trans. Wireless Commun., vol. 4, no. 2, pp. 341348, Mar. 2005.

[16] M. Y. Alias, S. Chen, and L. Hanzo, "Multiple-antenna-aided OFDM employing genetic-algorithm-assisted minimum bit error rate multiuser detection," IEEE Trans. Veh. Technol., vol. 54, no. 5, pp. 1713-1721, Sep. 2005.

[17] I. Lee, "The effect of a precoder on serially concatenated coding systems with an ISI channel," IEEE Trans. Commun., vol. 49, no. 7, pp. 11681175, Jul. 2001

[18] J. Hagenauer, E. Offer, and L. Papke, "Iterative decoding of binary block and convolutional codes," IEEE Trans. Inf. Theory, vol. 42, no. 2, pp. 429 445, Mar. 1996.

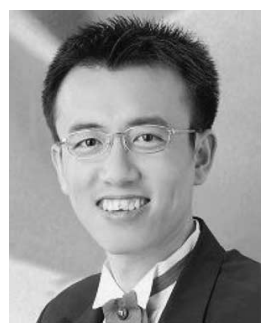

Shuang Tan received the B.E. and M.E. degrees in electronic information engineering from Beijing University of Aeronautics and Astronautics, Beijing, China, in 1999 and 2002, respectively.

$\mathrm{He}$ is currently with the School of Electronics and Computer Science, University of Southampton, Southampton, U.K.

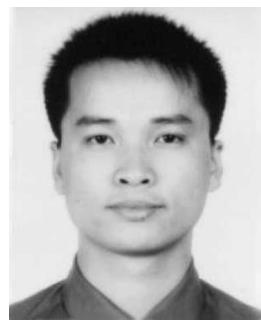

Jin Wang ( $\mathrm{S}^{\prime} 03$ ) received the B.E. degree from the University of Science and Technology of China, Hefei, China, in 1999 and the M.E. degree in video signal processing from the Graduate School of Chinese Academy of Sciences, Beijing, China, in 2002.

$\mathrm{He}$ is currently with the School of Electronics and Computer Science, University of Southampton, Southampton, U.K. 


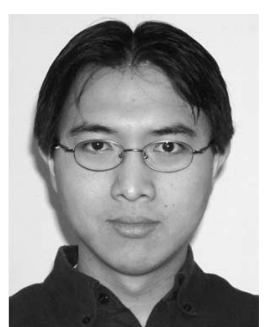

Soon Xin Ng (S'99-M'03) received the B.Eng. degree (first class) in electronics engineering and the $\mathrm{Ph} . \mathrm{D}$. degree in wireless communications from the University of Southampton, Southampton, U.K., in 1999 and 2002, respectively.

From 2003 to 2006, he was a Postdoctoral Research Fellow, working on collaborative European research projects such as SCOUT, NEWCOM, and PHOENIX. Since August 2006, he has been a Lecturer of wireless communications with the School of Electronics and Computer Science, University of Southampton.

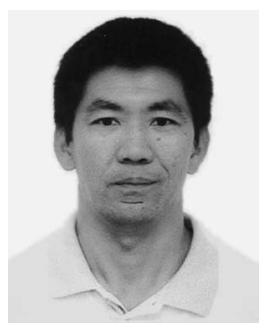

Sheng Chen (M'90-SM'97) received the B.Eng. degree in control engineering from East China Petroleum Institute, Dongying, China, in 1982, the Ph.D. degree in control engineering from the City University, London, U.K., in 1986, and the D.Sc. degree from the University of Southampton, Southampton, U.K., in 2005.

$\mathrm{He}$ is currently with the School of Electronics and Computer Science, University of Southampton.

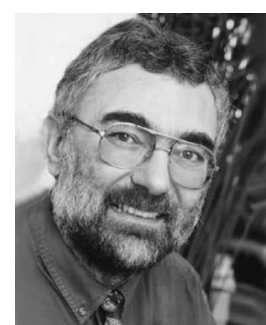

Lajos Hanzo (M'91-SM'92-F'04) received the M.S. degree in electronics and the Ph.D. degree from the Technical University of Budapest, Budapest, Hungary, in 1976 and 1983, respectively, and the D.Sc. degree from the University Southampton, Southampton, U.K., in 2004.

During his 30-year career in telecommunications, he has held various research and academic posts in Hungary, Germany, and U.K. Since 1986, he has been with the School of Electronics and Computer Science, University of Southampton, where he holds the Chair in Telecommunications. He is currently managing an academic research team, working on a range of research projects in the field of wireless multimedia communications sponsored by the industry, the Engineering and Physical Sciences Research Council, U.K., the European IST Program, and the Mobile Virtual Centre of Excellence, U.K. (for further information on research in progress and associated publications, see http://www-mobile.ecs. soton.ac.uk). He is an enthusiastic supporter of industrial and academic liaison and offers a range of industrial courses. He has coauthored 15 John Wiley/IEEE Press books totaling about 8000 pages on mobile radio communications, published more than 700 research papers, organized and chaired conference sessions, and presented overview lectures. He is the Editor-in-Chief of the IEEE Press.

Dr. Hanzo is a Fellow of the Royal Academy of Engineering and Institution of Electrical Engineers. He is a Distinguished Lecturer of the IEEE Communications Society. He is a Distinguished Lecturer and an elected Governor of the IEEE Vehicular Technology Society. He was a recipient of a number of distinctions. 